\title{
Particular Threshold Behavior of Dusty Plasma Instabilities
}

\author{
M. Mikikian*, M. Cavarroc, L. Couëdel, Y. Tessier and L. Boufendi \\ GREMI, UMR6606 CNRS-Université d'Orléans, 14 rue d'Issoudun, BP6744, 45067 Orléans Cedex 2, \\ France,*maxime.mikikian@univ-orleans.fr
}

\begin{abstract}
We show that some experimentally observed instabilities, concerning the void region of a dust cloud, are similar to oscillations obtained in chemical systems or neuronal dynamics. The time evolution of these instabilities follows a well-defined process particularly visible in the instability shape and frequency.
\end{abstract}

Keywords: Void, Instabilities, Dusty plasmas.

PACS: 52.27.Lw, 52.35.-g, 52.25.Gj, 05.45.-a

\section{INTRODUCTION}

In plasma reactors, dust clouds are often characterized by a central dust-free region named "void" $[1,2]$. These dust-free regions are considered to be maintained by an equilibrium between inward electrostatic and outward ion drag forces [1]. Under certain conditions, the void equilibrium can be disturbed, resulting in self-excited low frequency (a few tens of $\mathrm{Hz}$ ) oscillations of the void size. The obtained instability, consisting of successive contractions and expansions of the void, is named "heartbeat" $[3,4]$. The plasma and dust cloud behavior during this instability is relatively complex especially when the instability is about to end [5]. Indeed, this self-excited instability can stop by its own through an ending phase characterized by failed contractions that appear more and more numerous as the end approaches. Thus, the phenomena sustaining the instability evolve progressively until a new stable state is reached.

\section{EVIDENCE OF FAILED VOID CONTRACTIONS}

The experiments are performed in the PKE-Nefedov reactor [6] where dust particles are grown from material sputtering [7] in a radio-frequency discharge. Dense clouds of submicron dust particles are obtained with a typical void in their center. The oscillation of the void size and the ending phase of the heartbeat instability are brought to the light thanks to electrical and optical characterizations. As an example, the first two oscillations in Fig. 1(a) are typical of the signature of the heartbeat instability on the electrical signal. When the instability is about to end, transitions to regimes with failed contractions occur. It is well observed in Fig. 1(a) where an arrow indicates a transition to a regime with one failed contraction. Then, these transitions occur more 
and more frequently and each new regime is characterized by one (or more) additional failed peak (regimes with about 40 failed peaks have been observed). Figure 1(b) shows a typical transition between one and two failed peak regimes on optical signals.
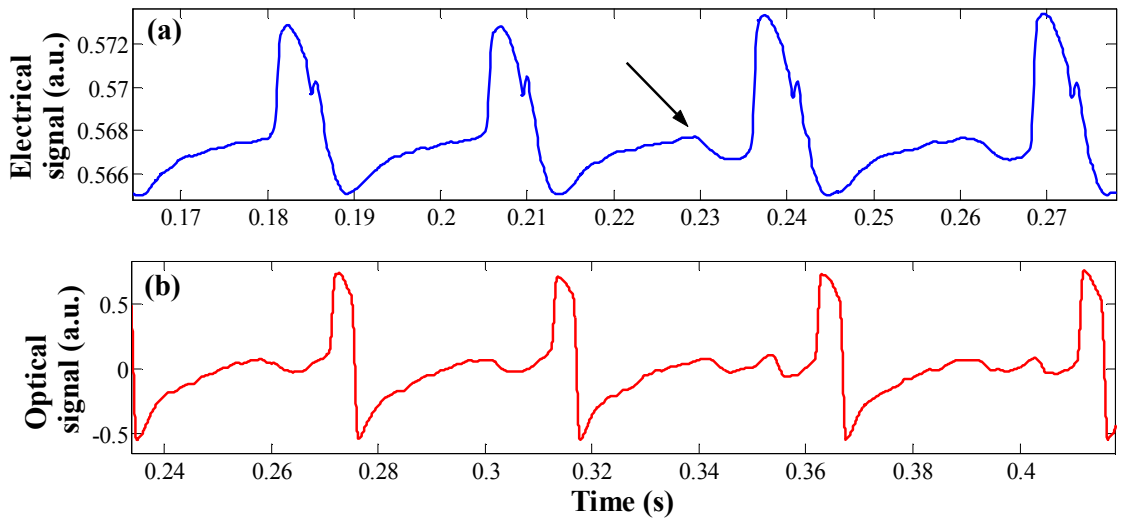

FIGURE 1. (a) Electrical signal with a transition from 0 to 1 failed peak. (b) Optical signal with a transition from 1 to 2 failed peaks.

It has been shown in [4] that void contractions correspond to an increase in the electrical and optical measurements. Indeed, at the moment indicated by an arrow in Fig. 1(a), a contraction (a drastic increase of the signal amplitude) is expected. Instead, a small decrease is observed and the contraction is delayed. To improve the experimental characterization of the instability, plasma emission in the whole discharge has been recorded using high speed imaging. In order to easily represent the time dependent plasma behavior, the central line of each frame has been extracted and all these lines have been placed side by side (Fig. 2). It clearly appears that bright plasma regions converge to the center during void reopening [4]. Then, the central emission starts increasing (meaning that the void is about to contract) but does not reach the conditions required for a new contraction. At this time a failed (or very small) contraction occurs (around image 188), which is confirmed by superimposing the corresponding electrical signal. A reopening takes place again and finally the real contraction occurs when the central plasma emission increases drastically.

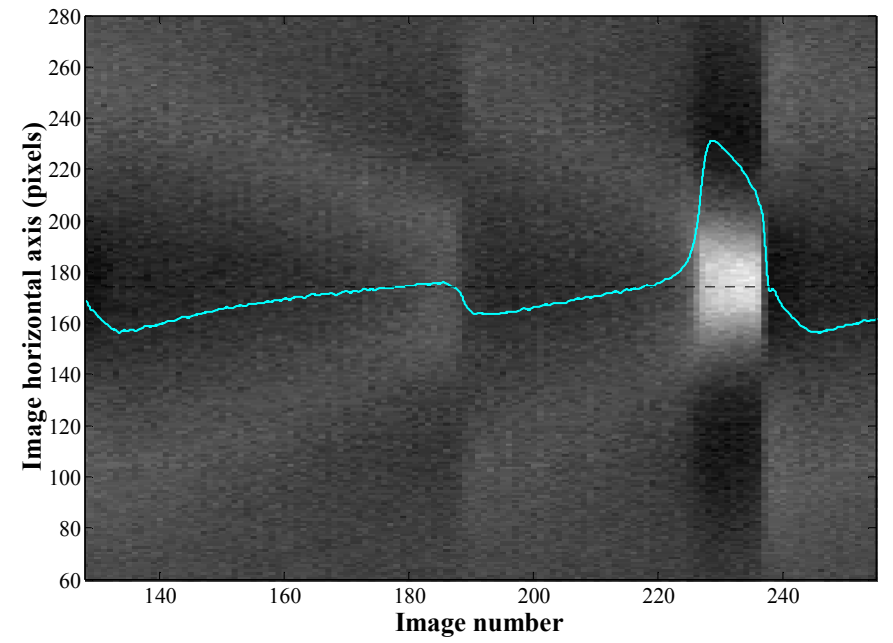

FIGURE 2. Image reconstruction of the plasma emission behavior during a regime with one failed peak. The corresponding electrical signal is superimposed 


\section{INSTABILITY ENDING PHASE}

Before the instability stops, more and more small amplitude failed peaks occur in between the high amplitude peaks. Thus, the apparent instability frequency (mainly defined by the high amplitude peaks) decreases. This behavior is well observed in Fig. 3 where the spectrogram of an ending phase is reported. The instability frequency is about $20 \mathrm{~Hz}$ and harmonics are observed. This spectrogram shows regular frequency steps characteristic of the occurrence of one additional failed peak at each transition. We can clearly observe that the duration of each regime decreases as the end approaches. Just before the end, regimes are short and several additional failed peaks can appear during one single transition. Then, the instability stops at about $17.7 \mathrm{~s}$ and the void remains open and stable.

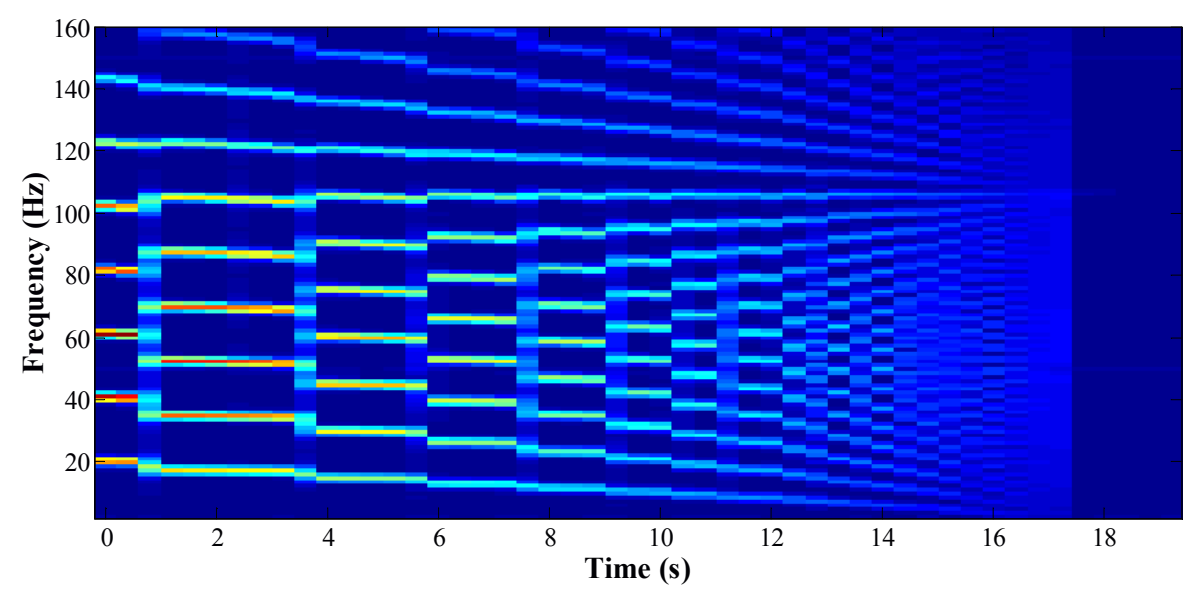

FIGURE 3. Spectrogram of an ending phase of the heartbeat instability

\section{MIXED-MODE OSCILLATIONS}

The observed signals in Fig. 1 have a well-defined shape that seems similar to mixed-mode oscillations (MMOs). These specific oscillations are complex phenomena consisting of an alternation of a varying number of small amplitude oscillations and large amplitude ones (also named spikes). Small and large amplitude oscillations are often considered as, respectively, subthreshold oscillations and relaxation mechanisms of the system. These MMOs are encountered in a wide variety of fields. In chemistry, reaction kinetics can take the form of MMOs like in the intensively studied BelousovZhabostinskii reaction [8]. In natural sciences, MMOs are the subject of an intense research since their observation in the Hodgkin-Huxley model of neuronal activity [9]. In this context, they are strongly related to spiking and bursting activities in neurons.

In the classical notation of MMOs, a pattern characterized by L high amplitude oscillations and $\mathrm{S}$ small amplitude ones will be noted $\mathrm{L}^{\mathrm{S}}$. As an example, Fig. 1(a) is a transition from $1^{0}$ to $1^{1}$ state, whereas Fig. 1(b) corresponds to a $1^{1}-1^{2}$ transition. As it was observed in Fig. 3, close to the end of the instability (after $15 \mathrm{~s}$ ), the system evolves faster and faster and transitions between $\mathrm{L}^{\mathrm{S}}$ and $\mathrm{L}^{\mathrm{S}+\mathrm{n}}$ states (with $\mathrm{n}$ increasing from 2 to 5) are observed. 
In neuronal activity and corresponding dynamical system theories, statistics on the interspike intervals (ISI) is often used. It corresponds to the time in between two large amplitude oscillations. Results are globally similar to the spectrogram analysis shown in Fig. 3 but can better bring to light some slight changes. Indeed, performing this analysis reveals that a transition between a $\mathrm{L}^{\mathrm{S}}$ and a $\mathrm{L}^{\mathrm{S}+1}$ state is the consequence of a slight but progressive increase in the interspike interval during the $\mathrm{L}^{\mathrm{S}}$ state. The ISIs of the $\mathrm{L}^{\mathrm{S}}$ state increase slowly until a new failed peak appears in between the spikes and thus the $\mathrm{L}^{\mathrm{S}+1}$ state is established. When conditions of the $\mathrm{L}^{\mathrm{S}+1}$ state are not perfectly met, the system can "hesitate" between the two states and can turn back to the previous $\mathrm{L}^{\mathrm{S}}$ state. Thus, situations with multiple "back and forth" between two states can be observed. Usually, these situations are characterized by the progressive occurrence of more and more $\mathrm{L}^{\mathrm{S}+1}$ states in between $\mathrm{L}^{\mathrm{S}}$ ones until conditions for a permanent $\mathrm{L}^{\mathrm{S}+1}$ regime are fulfilled.

\section{CONCLUSIONS}

In this work, we evidenced and characterized mixed-mode oscillations in dusty plasmas. This specific type of oscillation has been observed during the ending phase of the heartbeat instability which is characterized by an alternation of small and high amplitude oscillations. Small amplitude oscillations correspond to failed void contractions. This work highlights new situations of MMOs that can be of interest for improving dynamical system theories related to these structures. The obtained waveforms are very similar to what is observed in oscillating chemical systems and in neuronal activity. These fields use well-known sets of equations giving rise to MMOs. This scientific background can thus be used to explore and develop new theoretical approaches in dusty plasma dynamics.

\section{ACKNOWLEDGMENTS}

The PKE-Nefedov chamber has been made available by the Max-Planck-Institute for Extraterrestrial Physics, Germany, under the funding of DLR/BMBF under grants No.50WM9852. This work was supported by CNES under contract 02/CNES/4800000059.

\section{REFERENCES}

1. J. Goree, G.E. Morfill, V.N. Tsytovich, and S.V. Vladimirov, Phys. Rev. E 59, 7055 (1999)

2. M. Cavarroc, M. Mikikian, Y. Tessier and L. Boufendi, Phys. Rev. Lett. 100, 045001 (2008)

3. M. Mikikian and L. Boufendi, Phys. Plasmas 11, 3733 (2004)

4. M. Mikikian, L. Couëdel, M. Cavarroc, Y. Tessier and L. Boufendi, New J. Phys. 9, 268 (2007)

5. M. Mikikian, M. Cavarroc, L. Couëdel, Y. Tessier and L. Boufendi, submitted to Phys. Rev Lett.

6. A P. Nefedov et al., New J. Phys. 5, 33 (2003)

7. M. Mikikian et al. New J. Phys. 5, 19 (2003)

8. J.L. Hudson, M. Hart, and D. Marinko, J. Chem. Phys. 71, 1601 (1979)

9. J. Guckenheimer, R. Harris-Warrick, J. Peck, and A. Willms, J. Comput. Neurosci. 4, 257 (1997) 